\title{
Preparation and characterization of doxorubicin functionalized tiopronin-capped gold nanorods for cancer therapy
}

\author{
HUO ShuaiDong
LIANG XingJie $^{1,2 \dagger}$ \\ ${ }^{1}$ CAS Key Laboratory for Biological Effects of Nanomaterials and Nanosafety, National Center for Nanoscience and Technology, Beijing 100190, \\ China; \\ ${ }^{2}$ School of Materials Science and Engineering, Tianjin Polytechnic University, Tianjin 300387, China
}

Received November 28, 2012; accepted January 23, 2013; published online June 21, 2013

Gold nanomaterials are immerging candidates in medical diagnosis and treatment. Among them, gold nanorods (Au NRs) are widely used for cancer treatment. Tiopronin as a novel thiol drug was used to stabilize Au NRs in this work. Doxorubicin (DOX), a chemotherapeutic drug which works by interacting with DNA to arrest the cell cycle and induce apoptosis, was linked to Au NRs through electrostatic reaction with tiopronin, to obtain Au-TIOP-DOX NRs. Au NRs are also regarded as hyperthermia agents for photothermal cancer treatment. This delivery system (Au-TIOP-DOX NRs) was designed for passively targeting tumor cells in cancer therapy. More importantly, the carboxyl groups of tiopronin can be modified with some biological molecules (DNA/RNA, peptides, or drugs) to make Au NRs as a novel drug-delivery system for cancer treatment.

gold nanorods, tiopronin, doxorubicin, cancer therapy

Citation: Huo S D, Jin S B, Zheng K Y, et al. Preparation and characterization of doxorubicin functionalized tiopronin-capped gold nanorods for cancer therapy. Chin Sci Bull, 2013, 58: 4072-4076, doi: 10.1007/s11434-013-5918-8

Gold nanorods (Au NRs) are promising nanomaterials as therapeutics, imaging probes, contrast agents and drug vehicles in cancer treatment [1-4]. The unique properties, such as tunable surface plasmon resonance (SPR), twophoton luminescence and ease of functionalization, make them suitable in thermal therapy, cell imaging, gene silencing and targeted drug delivery [5-8]. PEGylated Au NRs are reported to be more stable in physiological condition and obtain a longer circulation time in vivo $[9,10]$. In addition, Dreaden et al. [11] synthesized a small molecule$\mathrm{Au}$ NRs conjugate which can selectively target tumorassociated macrophage cells and induce cytotoxicity to breast cancer cells.

Cetyltrimethylammonium bromide (CTAB), a cationic detergent, is used as stabilizer and surface coating in the preparation of $\mathrm{Au}$ NRs and it is highly toxic to cells. Due to the free CTAB in the Au NRs solution which can hardly be

\footnotetext{
$\dagger$ These authors contributed equally to this work.

*Corresponding authors (email: wangdl@nanoctr.cn; liangxj@nanoctr.cn)
}

removed totally, the biological application of Au NRs is limited because of the cell toxicity $[12,13]$. Much work has been done to optimize the surface coating of Au NRs and then to reduce the cytotoxicity, improve the biocompatibility and increase the drug loading efficiency [14-16]. Among these work, PEGylation seems to be a good choice as it could prolong the circulation time of $\mathrm{Au} \mathrm{NRs}$ in the circulatory system, escape from the clearance of reticuloendothelial system (RES) [17]. However, the cellular uptake of PEG-Au NRs is not that satisfactory to people's expectation and we are looking for a better surface coating of $\mathrm{Au}$ NRs. In this work, we replace the CTAB on the Au NRs surface with tiopronin, a novel thiol-group drug which could form $\mathrm{Au}-\mathrm{S}$ bond, a covalent bond stronger than the interaction between $\mathrm{CTAB}$ and $\mathrm{Au}$ atom. Thus, the replacement of CTAB is highly efficient and biocompatibility is improved. Tiopronin also has a carboxyl group which is capable of lots of chemical reactions; therefore, much therapeutics can be loaded on Au NRs. Here, we functionalized 
tiopronin-coated Au NRs with a clinical used cancer therapeutic, doxorubicin (DOX), and the treatment efficacy of Au-TIOP-DOX NRs were assessed initially $[18,19]$.

\section{Materials and methods}

(i) Chemicals. Gold chloride trihydrate $(\geqslant 99.9 \%$, $\left.\mathrm{HAuCl}_{4} \cdot 3 \mathrm{H}_{2} \mathrm{O}\right)$, cetyltrimethylammonium bromide $(\geqslant 98 \%$, CTAB, $\mathrm{C}_{19} \mathrm{H}_{42} \mathrm{BrN}$ ) and $\mathrm{N}$-(2-mercaptopropionyl) glycine (tiopronin, $\mathrm{C}_{5} \mathrm{H}_{9} \mathrm{NO}_{3} \mathrm{~S}$ ) were purchased from Sigma-Aldrich (St Louis, USA). L(+)-Ascorbic acid (99.0\%) was supplied by Acros (USA). Sodium citrate $\left(\geqslant 99.5 \%, \mathrm{C}_{6} \mathrm{H}_{5} \mathrm{Na}_{3} \mathrm{O}_{7} \cdot 2 \mathrm{H}_{2} \mathrm{O}\right)$ was obtained from Solarbio (Beijing, China). Silver nitrate $\left(\geqslant 99.8 \%, \mathrm{AgNO}_{3}\right.$ ) was purchased from GuangFu (Tianjin, China). Doxorubicin $(\geqslant 99 \%$, DOX) was purchased from Zhejiang Hisun Pharmaceutical Co. Ltd. All glass wares used for the preparation and storage of gold nanoparticles were cleaned with aqua regia $\left(\mathrm{HCl}: \mathrm{HNO}_{3}=3: 1\right.$, v:v). All chemicals were used without further purification and Milli-Q water was used throughout this study.

(ii) Synthesis of CTAB capped gold nanorods. CTAB capped Au NRs (Au-CTAB NRs) were prepared according to the seed-mediated growth method [20]. Briefly, a seed solution was prepared by mixing $9.15 \mathrm{~mL}$ of CTAB $(0.1$ $\mathrm{mol} / \mathrm{L})$ and $0.25 \mathrm{~mL}$ of $\mathrm{HAuCl}_{4}(0.01 \mathrm{~mol} / \mathrm{L})$ with $0.6 \mathrm{~mL}$ freshly prepared ice-cold $\mathrm{NaBH}_{4}$ solution $(0.1 \mathrm{~mol} / \mathrm{L})$. The color of the solution changed from dark yellow to brownish yellow under vigorous stirring, indicating the formation of the seed solution. This seed solution was used within 2-6 h for the synthesis of the Au-CTAB NRs. Briefly, $28.27 \mathrm{~mL}$ of $0.1 \mathrm{~mol} / \mathrm{L}$ CTAB was mixed with $1.2 \mathrm{~mL}$ of $0.01 \mathrm{~mol} / \mathrm{L}$ $\mathrm{HAuCl}_{4}$ and $0.18 \mathrm{~mL}$ of $0.01 \mathrm{~mol} / \mathrm{L}$ silver nitrate aqueous solution. After gentle mixing of the solution, $0.2 \mathrm{~mL} 0.1$ $\mathrm{mol} / \mathrm{L}$ ascorbic acid was added, and then the solution changed to colorless transparent. With continuously stirring, $0.15 \mathrm{~mL}$ of the seed solution was added to initiate the growth of the Au-CTAB NRs.

(iii) Tiopronin coating of Au NRs. A surface ligand exchange reaction was adopted to obtain tiopronin coating of Au NRs (Au-TIOP NRs). Briefly, $1 \mathrm{~mL}$ of Au-CTAB NRs was centrifuged at $9000 \mathrm{r} / \mathrm{min}$ for $10 \mathrm{~min}$, and the precipitate was re-dispersed in $1 \mathrm{~mL}$ deionized water. Under vigorous stirring, $1 \mathrm{~mL}$ Au-CTAB NRs and $1 \mathrm{~mL}$ tiopronin solution $(2 \mathrm{mg} / \mathrm{mL})$ were mixed, sonicated for $60 \mathrm{~s}$ and stirred overnight at room temperature. The resulting $\mathrm{Au}$ NRs were then collected by centrifugation at $9000 \mathrm{r} / \mathrm{min}$ for 15 min and the tiopronin coated Au NRs were obtained.

(iv) Preparation of DOX-conjugated Au NRs. DOX loading onto Au-TIOP NRs was done by simply mixing 1 $\mathrm{mL} 0.5 \mathrm{mg} / \mathrm{mL}$ DOX with $1 \mathrm{~mL}$ Au-TIOP NRs. The resulting solution was kept stirring overnight at room temperature. Excess DOX molecules were removed by centrifugation at $10000 \mathrm{r} / \mathrm{min}$ for $10 \mathrm{~min}$. To evaluate the DOX-loading efficiency, the content of the residual DOX in the supernatant was determined by UV-Vis measurements at $480 \mathrm{~nm}$ [18]. The DOX-loading efficiency was calculated by

$$
\begin{aligned}
& \text { Loading efficiency }(\%) \\
& =\frac{m(\text { total DOX })-m(\text { DOX in supernatant })}{m(\text { total DOX })} \times 100 \% .
\end{aligned}
$$

(v) Cell culture. The human breast cancer cell line MCF-7 was maintained in Dulbecco's modified Eagle's medium (DMEM) with $10 \%$ fetal bovine serum in a humidified atmosphere containing $5 \% \mathrm{CO}_{2}$ at $37^{\circ} \mathrm{C}$.

(vi) Cell viability assay. 3-(4,5-dimethylthiazol-2-yl)2,5-diphenyltetrazolium bromide (MTT) assay was used to evaluate the viability of MCF-7 cells. MCF-7 cells were seeded in 96-well plate, and after $24 \mathrm{~h}$, Au-TIOP-DOX NRs and free DOX were added at the concentration of $0.08,0.4$, $2,10 \mu \mathrm{g} / \mathrm{mL}$ based on DOX into the plate. As negative controls, cells were cultured with Au-CTAB NRs and Au-TIOP $\mathrm{NRs}$ at the corresponding concentration of Au NRs with the Au-TIOP-DOX NRs added. Another $24 \mathrm{~h}$ later, $10 \mu \mathrm{L}$ MTT solution was added per well, and after $4 \mathrm{~h}$ of incubation, formazan crystals were dissolved in $150 \mu \mathrm{L}$ DMSO. The absorbance was measured at $570 \mathrm{~nm}$ with a reference wavelength of $630 \mathrm{~nm}$ using an Infinite M200 microplate reader (Tecan, Durham, NC, USA).

(vii) Characterization. Morphology characterization of the Au NRs was performed using a Tecnai $\mathrm{G}^{2} 20$ S-TWIN transmission electron microscope (TEM, Philips, Netherlands) with $200 \mathrm{kV}$ acceleration voltage. Optical absorption spectra were measured with a Lambda $950 \mathrm{UV} / \mathrm{Vis} / \mathrm{NIR}$ spectrophotometer (Perkin-Elmer, USA). Zeta potential distribution was determined by a Nano ZS Zetasizer (Malvern, England). The concentration of gold was determined via an Optima 5300DV Inductively Coupled Plasma Optical Emission Spectrometer, or an ELAN DRC-e Inductively Coupled Plasma Mass Spectrometer (Perkin-Elmer).

\section{Results and discussion}

The preparation of DOX-conjugated Au NRs (Au-TIOPDOX NRs) is shown in Figure 1. CTAB stabilized Au NRs (Au-CTAB NRs) with aspect ratios $\sim 4$ were prepared firstly using a seed-growth method, the most popular method for the synthesis of colloidal Au NRs [20]. Tiopronin stabilized $\mathrm{Au}$ NRs (Au-TIOP NRs) were obtained via a welldeveloped ligand exchange method [21]. As we all know, thiol group exchange is the most common way to replace the original capping molecules since the metal-sulfur bond is known to be the strongest bond compared to other general functional groups. Tiopronin is a thiol drug with good biocompatibility used to control the rate of cysteine precipitation and excretion in the disease named cystinuria [22,23]. It is commonly used as a stabilizing agent for metal nanoparticles, as its thiol groups can bind to the surface of nanoparticles, preventing aggregation and sedimentation 


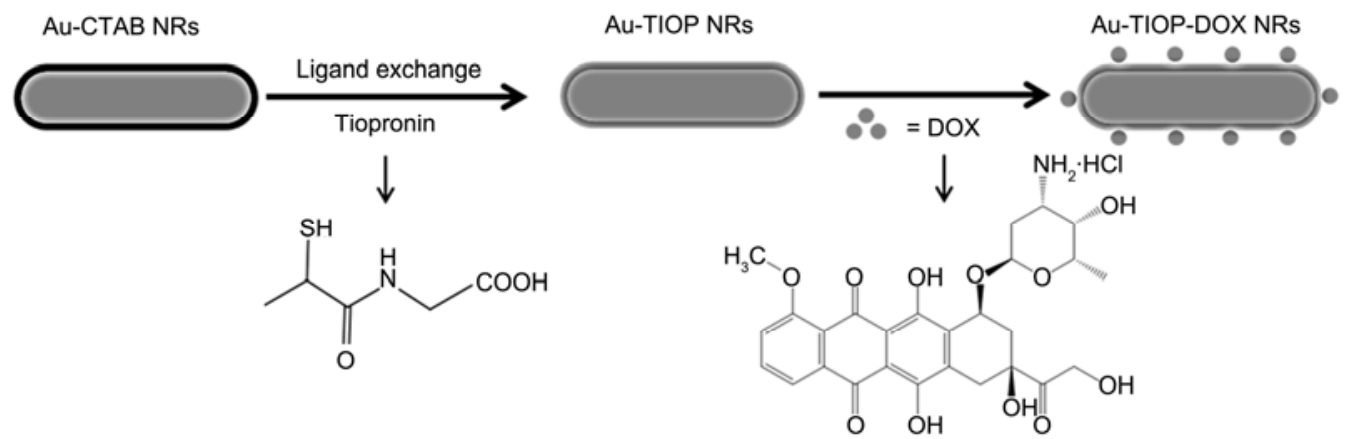

Figure 1 Illustration of the procedure for preparing the doxorubicin functionalized tiopronin-capped Au NRs.

[24]. In this work, tiopronin not only makes Au NRs stable in various physiological relevant media but also makes the outside of the nanorods bearing carboxyl groups. The resulting Au-TIOP NRs exhibited reversed surface charge and allowed for the electrostatic adsorption of DOX.

As shown in Figure 2, the transmission electron microscopy (TEM) images showed Au NRs in each step with a uniform morphology with aspect ratios $\sim 4$. It is also found that compared with CTAB capped Au NRs, Au-TIOP NRs have no change on shape and size after ligand-exchange reaction. From the negative staining TEM images of $\mathrm{Au}$-TIOP NRs and Au-TIOP-DOX NRs shown in Figure 3, after DOX absorption reaction, an about $2 \mathrm{~nm}$ thin coating can be seen around the surface of Au-TIOP-DOX.

In order to confirm each step of the surface-modification of Au NRs, zeta potential study was conducted. As shown in Figure 4, the zeta potential value of the as-prepared Au-CTAB NRs is $+30.5 \mathrm{mV}$. After ligand exchange reaction with tiopronin, the value changes to $-18.9 \mathrm{mV}$, indicating the charge reverse of the Au NRs because of the free negatively charged carboxy groups of tiopronin exposed on
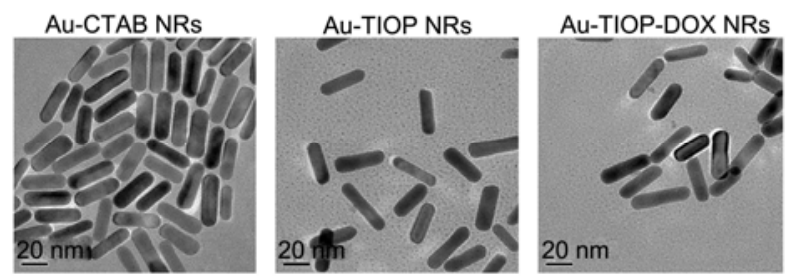

Figure 2 TEM images of Au-CTAB NRs, Au-TIOP NRs and Au-TIOP-DOX NRs.
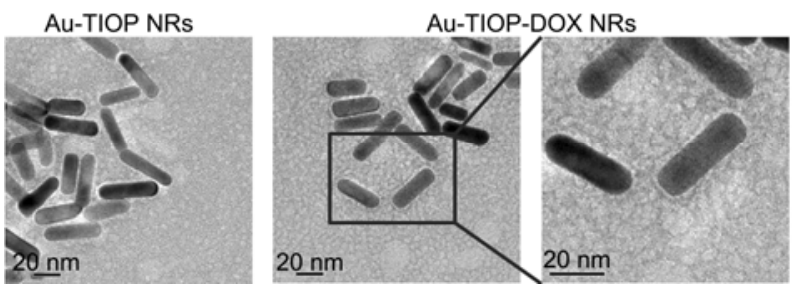

Figure 3 Negative stained TEM images of Au-TIOP NRs and Au-TIOP-DOX NRs. the outside surface of Au NRs. The successful adsorption of DOX makes the zeta potential to a value of $-3.6 \mathrm{mV}$. Meanwhile, the photos in Figure 4 showed the color change in each step of different capped Au NRs. Moreover, after DOX binding to Au-TIOP NRs, the zeta potential remains a little negative, which may diffuse more quickly and perform better when delivering drugs deep into tissues [25].

In addition, to further determine whether the Au-TIOPDOX NRs is successfully obtained, the UV-Vis absorption spectra of Au NRs with different coatings in each step were carried out. Au NRs showed two absorption bands: a strong longitudinal band in the near-infrared region corresponding to electron oscillation along the long axis and a weak transverse band, similar to that of gold nanospheres, in the visible region corresponding to electron oscillations along the short axis [26-28]. Compared with CTAB capped Au NRs, the absorption peak of Au-TIOP NRs shifted from 734 to $744 \mathrm{~nm}$ as shown in Figure 5. After electrostatic adsorption of DOX, Au-TIOP-DOX NRs showed two absorption peaks at 480 and $760 \mathrm{~nm} ; 480 \mathrm{~nm}$ is the absorption peak of free DOX, and this demonstrated the attachment of DOX on the surface of Au-TIOP NRs. Because of the absorbance at 480 $\mathrm{nm}$, Au-TIOP-DOX NRs can be utilized as fluorescence probe for imaging. Current plasmonic nanoparticles for

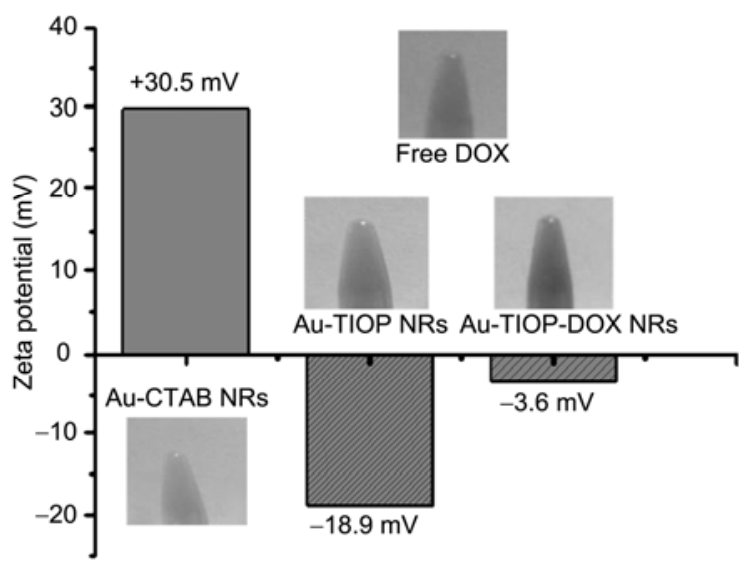

Figure 4 Zeta potential of Au-CTAB NRs, Au-TIOP NRs and $\mathrm{Au}-\mathrm{TIOP}-\mathrm{DOX}$ NRs. Corresponding pictures were shown on or under the zeta potential columns. 


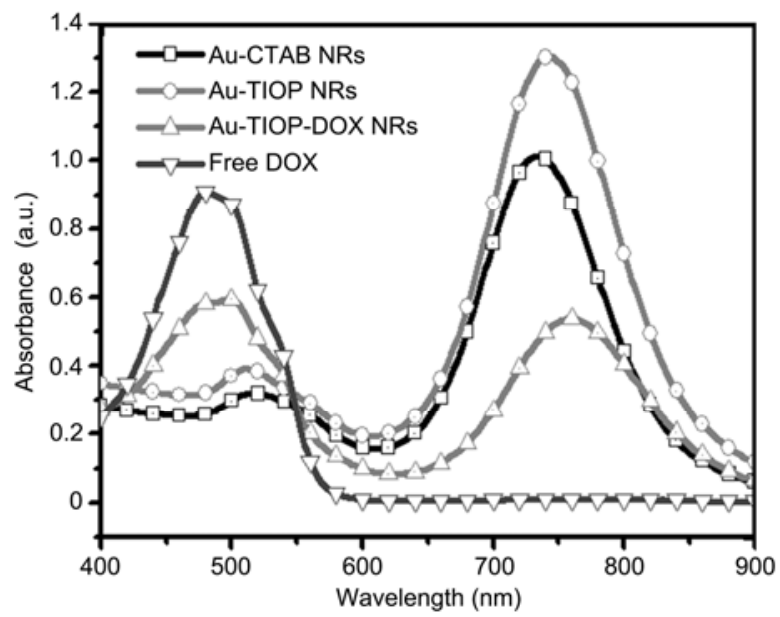

Figure 5 UV-Vis absorption spectra of Au-CTAB NRs, Au-TIOP NRs, Au-TIOP-DOX NRs and free DOX.

photothermal therapy of cancer are gold nanospheres, nanorods, nanoshells, and nanocages [3]. The last three types of plasmonic nanoparticles are more popular as they absorb light in the NIR window (650-900 nm [29]) where light penetration is optimal due to the minimal absorption by water and hemoglobin in the tissue. For their facile synthesis, excellent stability, small size, high photothermal conversion efficiency and tunable absorption in the biological window, Au NRs have become one of the most favorite nanoparticles for photothermal therapy. As UV-Vis absorption spectra shown in Figure 5, Au-TIOP-DOX NRs have a strong longitudinal band in the near-infrared region, so they also meet the conditions used for photothermal therapy.

In order to ensure sufficient DOX-loading onto the surface of gold nanorods by electrostatic adsorption, excess of DOX was added into the reaction. To evaluate the DOX-loading efficiency, the content of the residual DOX in the supernatant was determined by UV-Vis measurements at $480 \mathrm{~nm}$ [18]. The DOX-loading efficiency was calculated by eq. (1) is about $20 \%$ and the DOX-loading concentration is about $130 \mu \mathrm{g} / \mathrm{mL}$.

To evaluate the cell toxicity of each different capped $\mathrm{Au}$ NRs, 3-(4,5-dimethylthiazol-2-yl)-2,5-diphenylte trazolium bromide (MTT) assay was used to measure the cell viability after incubation with these nanomaterials. As shown in Figure 6, MCF-7 cells were cultured with Au-CTAB NRs, Au-TIOP NRs, Au-TIOP-DOX NRs and free DOX at different concentrations. The results showed that the cytotoxicity of Au-TIOP NRs is the lowest one, indicating the successful exchange of CTAB. Due to the free CTAB in the Au NRs solution which can hardly be removed totally, the Au-CTAB NRs had relatively stronge cell toxicity. The toxicity test indicated the replacement of CTAB was highly efficient. Compared with DOX, the Au-TIOP-DOX NRs had a better effect in killing cancer cells from the cell viability test. The high cytotoxic effect of Au-TIOP-DOX NRs is considered as a result of the release of DOX into MCF-7

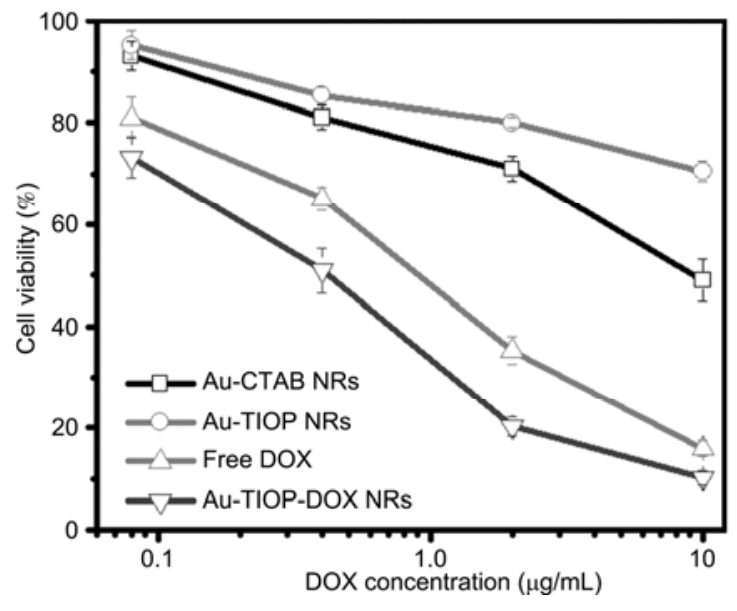

Figure 6 Cell viability of MCF-7 cells after $24 \mathrm{~h}$ treatment.

cells after the cellular uptake. Hence, we expect that $\mathrm{Au}$-TIOP-DOX NRs have promising potential as drug loading and delivery carriers in cancer therapy.

\section{Conclusions}

In summary, Au-TIOP-DOX NRs were prepared through thiol exchange and electrostatic adsorption method. Tiopronin, a thiol drug with good biocompatibility, was used to replace the CTAB on the surface of Au NRs, and the reversed surface charge of Au-TIOP NRs allowed for the further electrostatic adsorption of DOX. Characterizations were carried out to verify each step of different coatings of $\mathrm{Au}$ NRs. Furthermore, the cell viability was tested to determine the cancer-killing effect and the result was that the Au-TIOP-DOX NRs have a better cytotoxicity compared to free DOX. More importantly, the carboxyl groups of tiopronin not only can be used for electrostatic adsorption but also can be modified with molecules, peptides or drugs for active targeting purposes. The prepared doxorubicin functionalized tiopronin-capped $\mathrm{Au}$ NRs can be promising in drug-delivery, fluorescence imaging and localized hyperthermia probe for cancer therapy.

This work was supported by the National Natural Science Foundation of China (30970784, 81171455 and 31100720), National Basic Research Program of China (2009CB930200), Chinese Academy of Sciences (CAS) "Hundred Talents Program" (07165111ZX), CAS Knowledge Innovation Program and Tianjin Research Program of Applied Basic \& Cutting-edge Technologies (09JCYBJC27200). We also thank for the support from the Joint Laboratory of Nanotechnology for Bioapplication, which was established with Life Technologies Corp. in the National Center for Nanoscience and Technology of China.

1 Agarwal A, Huang S W, O'Donnell M, et al. Targeted Au NR contrast agent for prostate cancer detection by photoacoustic imaging. J Appl Phys, 2007, 102: 064701

2 Hauck T S, Jennings T L, Yatsenko T, et al. Enhancing the toxicity of cancer chemotherapeutics with Au NR hyperthermia. Adv Mater, 
2008, 20: 3832-3838

3 Guo R, Zhang L, Qian H, et al. Multifunctional nanocarriers for cell imaging, drug delivery, and near-IR photothermal therapy. Langmuir, 2010, 26: 5428-5434

4 Xie S S. Preface to the special topic on Nanotechnology for Bio/Energy Applications. Sci China Phys Mech Astron, 2012, 55: 1147

5 Bonoiu A C, Mahajan S D, Ding H, et al. Nanotechnology approach for drug addiction therapy: Gene silencing using delivery of $\mathrm{Au}$ NR-siRNA nanoplex in dopaminergic neurons. Proc Natl Acad Sci USA, 2009, 106: 5546-5550

6 Durr N J, Larson T, Smith D K, et al. Two-photon luminescence imaging of cancer cells using molecularly targeted Au NRs. Nano Lett, 2007, 7: 941-945

7 Huang X, El-Sayed I H, Qian W, et al. Cancer cell imaging and photothermal therapy in the near-infrared region by using Au NRs. J Am Chem Soc, 2006, 128: 2115-2120

8 Xiao Y, Hong H, Matson V Z, et al. Au NRs conjugated with doxorubicin and cRGD for combined anticancer drug delivery and PET imaging. Theranostics, 2012, 2: 757-768

9 Lankveld D P K, Rayavarapu R G, Krystek P, et al. Blood clearance and tissue distribution of PEGylated and non-PEGylated Au NRs after intravenous administration in rats. Nanomedicine, 2011, 6: 339-349

10 von Maltzahn G, Park J H, Agrawal A, et al. Computationally guided photothermal tumor therapy using long-circulating Au NR antennas. Cancer Res, 2009, 69: 3892-3900

11 Dreaden E C, Mwakwari S C, Austin L A, et al. Small molecule-Au NR conjugates selectively target and induce macrophage cytotoxicity towards breast cancer cells. Small, 2012, 8: 2819-2822

12 Alkilany A M, Nagaria P K, Hexel C R, et al. Cellular uptake and cytotoxicity of Au NRs: Molecular origin of cytotoxicity and surface effects. Small, 2009, 5: 701-708

13 Connor E E, Mwamuka J, Gole A, et al. Gold nanoparticles are taken up by human cells but do not cause acute cytotoxicity. Small, 2005, 1: 325-327

14 Takahashi H, Niidome Y, Niidome T, et al. Modification of Au NRs using phosphatidylcholine to reduce cytotoxicity. Langmuir, 2006, 22 $2-5$

15 Niidome T, Yamagata M, Okamoto Y, et al. PEG-modified Au NRs with a stealth character for in vivo applications. J Control Release, 2006, 114: 343-347
16 Li Z, Huang P, Zhang X, et al. RGD-conjugated dedrimer-modified $\mathrm{Au}$ NRs for in vivo tumor targeting and photothermal therapy. Mol Pharm, 2009, 7: 94-104

17 Akiyama Y, Mori T, Katayama Y, et al. The effects of PEG grafting level and injection dose on $\mathrm{Au}$ NR biodistribution in the tumorbearing mice. J Control Release, 2009, 139: 81-84

18 Wang T T, Zhang X L, Pan Y, et al. Fabrication of doxorubicin functionalized gold nanorod probes for combined cancer imaging and drug delivery. Dalton Trans, 2011, 40: 9789-9794

19 Mirza A Z, Shamshad H. Preparation and characterization of doxorubicin functionalized gold nanoparticles. Eur J Med Chem, 2011, 46: 1857-1860

20 Sau T K, Murphy C J. Seeded high yield synthesis of short Au nanorods in aqueous solution. Langmuir, 2004, 20: 6414-6420

21 Wijaya A, Hamad-Schifferli K. Ligand customization and DNA functionalization of $\mathrm{Au}$ NRs via round-trip phase transfer ligand exchange. Langmuir, 2008, 24: 9966-9969

22 Goldsborough A S, Handley M D, Dulcey A E, et al. Collateral sensitivity of multidrug-resistant cells to the orphan drug tiopronin. J Med Chem, 2011, 54: 4987-4997

23 Qiang F W, Wei P J, Li L. The effective propagation constants of SH wave in composites reinforced by dispersive parallel nanofibers. Sci China Phys Mech Astron, 2012, 55: 1172-1177

24 Huo S, Ma H, Huang K, et al. Superior penetration and retention behavior of $50 \mathrm{~nm}$ gold nanoparticles in tumors. Cancer Res, 2013, 73: 319-330

25 Kim B, Han G, Toley B J, et al. Tuning payload delivery in tumour cylindroids using gold nanoparticles. Nat Nanotech, 2010, 5: 465-472

26 Link S, Mohamed M, El-Sayed M. Simulation of the optical absorption spectra of Au NRs as a function of their aspect ratio and the effect of the medium dielectric constant. J Phys Chem B, 1999, 103: 3073-3077

27 Ji R N, Ye Y X, Hu X Y, et al. The green-emitting fluorescence of nano $\mathrm{Y}_{2} \mathrm{O}_{3}: \mathrm{Er}^{3+}$ under different excitations. Sci China Phys Mech Astron, 2012, 55: 1152-1157

28 Wang H Y, Feng Y K, Yuan W J, et al. Fabrication and characterization of electrospun biocompatible PU/PEGMA hybrid nanofibers by in-situ UV photopolymerization. Sci China Phys Mech Astron, 2012, 55: 1189-1193

29 Weissleder R. A clearer vision for in vivo imaging. Nat Biotech, 2001 19: $316-331$

Open Access This article is distributed under the terms of the Creative Commons Attribution License which permits any use, distribution, and reproduction in any medium, provided the original author(s) and source are credited. 